\title{
Influence of substituents on the energy and nature of the lowest excited states of heteroleptic phosphorescent Ir(III) complexes: A joint theoretical and experimental study
} Igor Avilov ${ }^{1}$, Payam Minoofar ${ }^{2}$, Jérôme Cornil ${ }^{1, \#}$, Luisa De Cola ${ }^{2}$

${ }^{1}$ Service de Chimie des Matériaux Nouveaux, Université de Mons-Hainaut, Place du Parc 20, B-7000 Mons, Belgium

\footnotetext{
${ }^{2}$ Westfälische Wilhelms Universität Münster, Mendelstrasse 7, D-48149 Münster, Germany
} 


\section{Supporting information}

The supporting information contain:

- Table 1 describing the pairs of "natural transition orbitals" contributing the most to the description of the lowest triplet excited states of complexes 1-7.

- The $\mathrm{x}, \mathrm{y}, \mathrm{z}$ coordinates of complexes 1-7 in various states as obtained at the DFT level. 
Table 1. Contour plots of the pairs of "natural transition orbitals" contributing the most to the description of the lowest triplet excited states of complexes 1-7. The energies of the states are reported in the second column. The $\lambda$ value quantifying the contribution of the NTO pair is reported in the last column. "CI" corresponds to the sum of the squared CI coefficients associated to all singly excited configurations contributing to the excited state.

\begin{tabular}{|l|l|l|l|l|}
\hline $\begin{array}{l}\text { Excited } \\
\text { state }\end{array}$ & E, eV & Occupied NTO Unoccupied NTO & $\lambda$ / CI \\
\hline$T_{1}$ & 2.59 & &
\end{tabular}


Table 3 continued

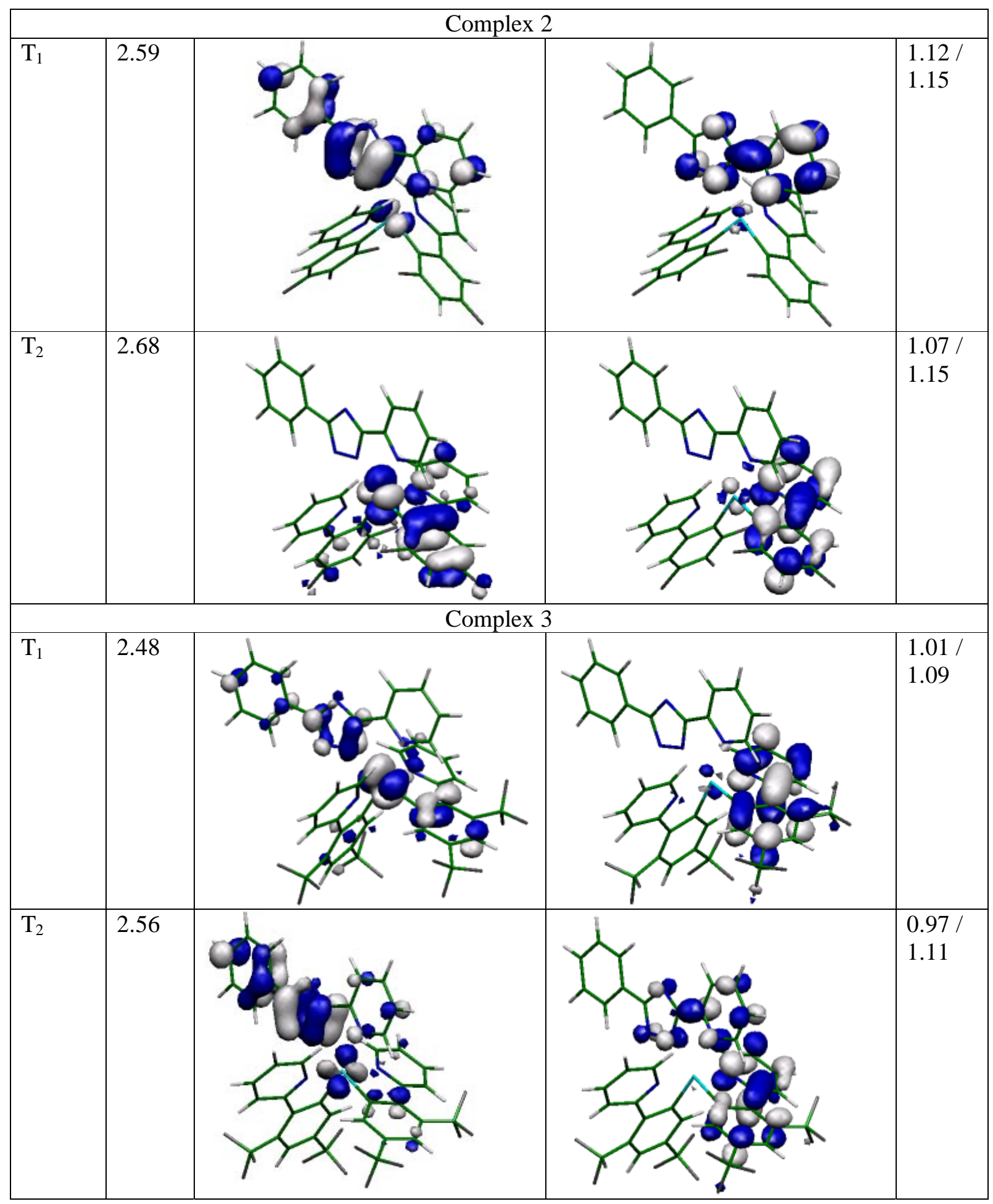


Table 3 continued

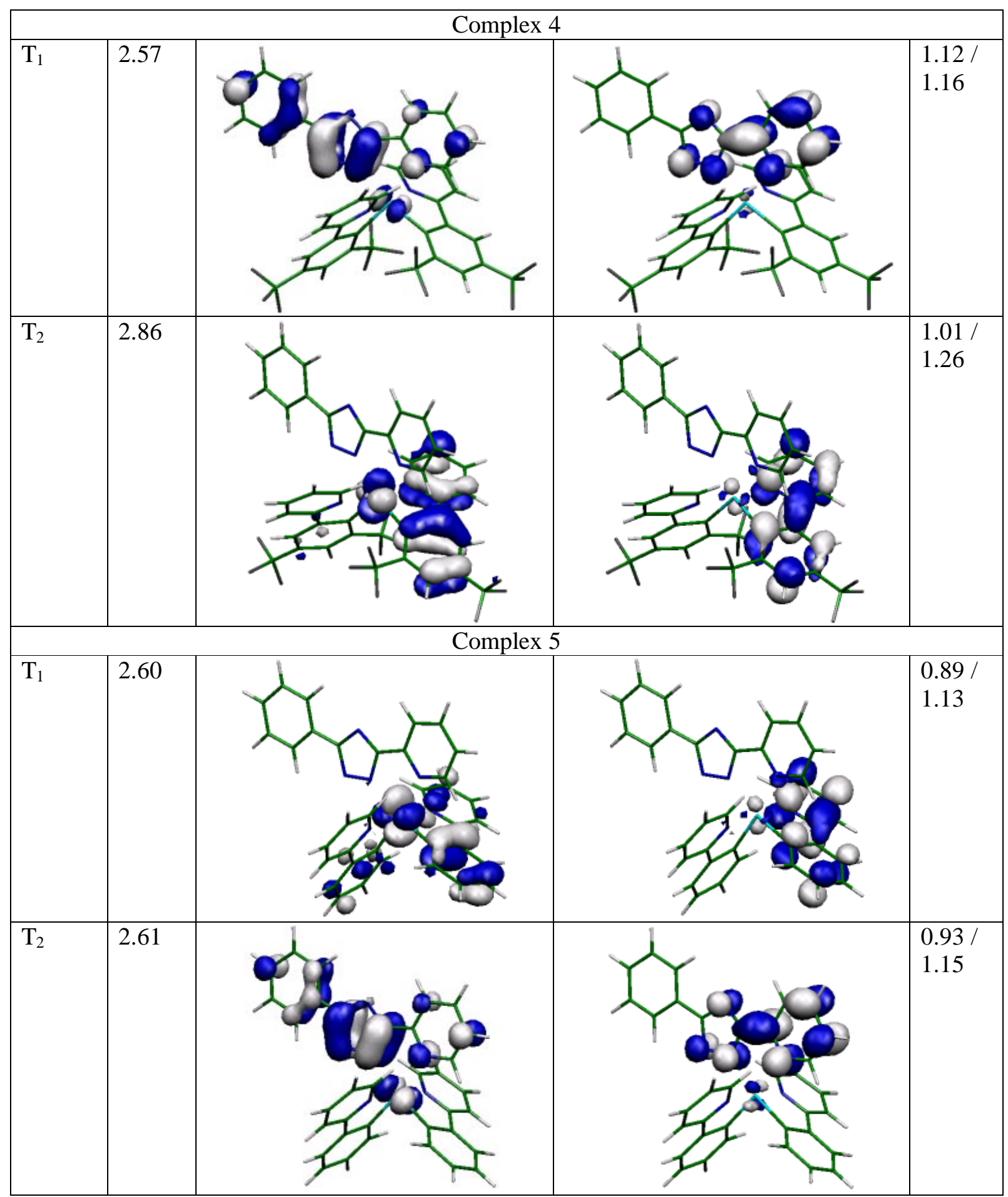


Table 3 continued

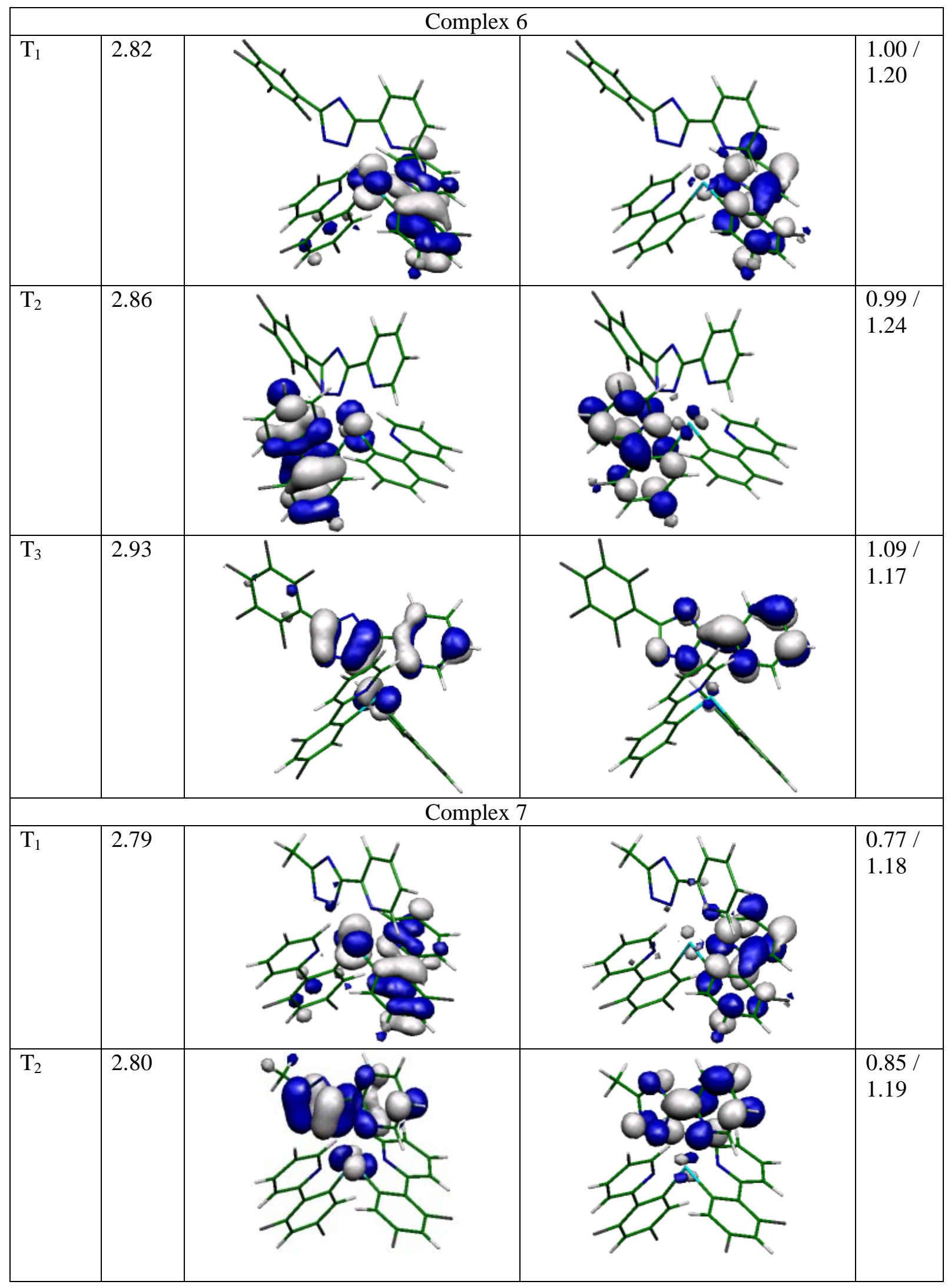


Complex 1 (S0)

Ir $0.90883170 .0207863-0.1117712$ C $2.6215477-1.11200472 .0058788$ C $3.5456617-0.60356870 .9924968$ C $4.9448106-0.68263571 .0505508$ C $5.7807286-0.17013570 .0711618$ C $5.16189790 .4509143-1.0108572$ C $3.78232670 .5642402-1.1364372$ C 0.86202671 .89168030 .6496118

C $0.62840172 .9162342-0.3205972$ C $0.48932972 .4654562-1.6998172$ C $0.24509373 .2653942-2.8322473$ C $0.14320172 .6826074-4.0923293$ C $0.28562171 .2959242-4.2256792$ C $0.52254170 .5398842-3.0843632$ N $0.62432171 .1005123-1.8540582$ $\mathrm{N}-1.1848483-0.3583388-0.0630242$ C $-1.5904833-1.5479887-0.6002412$ C $-0.5966012-2.4496787-1.1194602$ C $-0.8885323-3.7103577-1.6630362$ C $0.1536287-4.5079988-2.1273122$

C $1.4735937-4.0365909-2.0425882$

C $1.7058227-2.7772186-1.4950482$ N $0.6975237-1.9912258-1.0431832$ H $6.8553646-0.24749870 .1512078$ H $3.37756871 .0662133-2.0063052$ C $2.9554377-1.74097583 .2189138$ C $1.9502547-2.13780674 .0989978$ C $0.6099837-1.90323763 .7704117$ C $0.3172187-1.28412172 .5599358$ H -0.6956043 -1.0610468 2.2553477 H $2.2113778-2.61828675 .0354917$ H $2.3071877-4.6330755-2.3928902$ H - $0.0530213-5.4855807-2.5491562$ H - $1.9235382-4.0261376-1.7020272$ H $2.7028108-2.3657528-1.4077692$ $\mathrm{N}-2.9328773-1.7025297-0.5295912$ C -3.3377013 - 0.54201570 .0807248 $\mathrm{N}-2.29810020 .30108230 .3812358$ C $-4.7319572-0.22376580 .3826168$ C $-5.7519562-1.13468470 .0511428$ C - $7.0856821-0.83490570 .3370368$ C -7.42052620 .37575530 .9570118$ C -6.4088905 1.2861813 1.2893188 C -5.07368010.99153421.0053808 H -4.2858374 1.69203821 .2578888 H $0.21135370 .8076262-5.1894863$ H - $0.04692233 .3028023-4.9612963$ H $0.13656174 .3311464-2.7061992$ H $0.6352417-0.5335608-3.1265562$ C 0.54638674 .24637150 .1172338 C 0.68441174 .62409131 .4419508 C 0.91675573 .60012532 .3566167 
C 1.01082272 .26241131 .9962448 H 1.18983971 .52877922 .7704918 F $0.31659575 .2774493-0.8041742$ H 0.61528775 .65913931 .7433728 F 1.06398273 .94719623 .6958778 H -5.4809992 -2.0681427 -0.4288792 H -7.8645232 -1.5451928 0.0761558 $\mathrm{H}-6.66097622 .22691821 .7693218$ H -8.4579050 0.60748321 .1783048 H $3.9949317-1.90137963 .4588368$ H - $0.1957213-2.18716084 .4358966$ C $2.94105580 .0387973-0.1381292$ N $1.2925257-0.90580281 .6964698$ F $5.97550470 .9740453-2.0109172$ F 5.5715096 -1.3104158 2.1362908

Complex 1 (triplet; ppy-localized)

Ir $0.91906430 .0513592-0.1036086$

C $2.7410292-1.08954281 .9268775$

C $3.5798332-0.57472380 .9281754$

C $5.0158534-0.60403980 .9143034$

C $5.7713365-0.0729258-0.0947636$

C $5.09883640 .5439592-1.1773847$

C $3.71388530 .6270652-1.2486766$

C 0.84564831 .89871230 .6900284

C $0.57052232 .9366932-0.2552576$

C $0.39767932 .5140173-1.6412646$

C $0.09743733 .3332693-2.7452536$

C - $0.05321672 .7710361-4.0104967$

C $0.09474631 .3886212-4.1760545$

C $0.39242930 .6142272-3.0607706$

N $0.54633731 .1564212-1.8292566$

$\mathrm{N}-1.1736377-0.3791398-0.0345956$

C - $1.5621357-1.5825068-0.5523836$

C - $0.5594017-2.4761148-1.0712727$

C $-0.8370127-3.7539208-1.5809466$

C $0.2124593-4.5404527-2.0483196$

C $1.5238683-4.0408900-2.0033727$

C $1.7406513-2.7638378-1.4915866$

N $0.7251003-1.9913027-1.0364336$

H $6.8516092-0.1190648-0.0643286$

H $3.26457331 .1265002-2.0980826$

C $3.1120004-1.74664083 .1505863$

C $2.1573523-2.18685084 .0317926$

C $0.7707813-1.98121383 .7281634$

C $0.4193853-1.34287572 .5450284$

H - $0.6153538-1.15272682 .2913875$

H $2.4442522-2.67825684 .9540743$

H $2.3619693-4.6290319-2.3568216$

H $0.0176063-5.5319917-2.4422536$

H - $1.8655847-4.0919769-1.5911895$

H $2.7287883-2.3254037-1.4392906$ 
$\mathrm{N}-2.9024907-1.7492287-0.4866696$

C - $3.3243118-0.58124670 .0983854$

$\mathrm{N}-2.29634080 .27892920 .3874654$

C $-4.7239185-0.27378380 .3863864$

C $-5.7307339-1.20312780 .0661154$

C - $7.0697518-0.91373980 .3375244$

C -7.42284160.30464020.9316434

C -6.42427451 .23318831 .2531215$

C -5.08385850.94896820.9836444

H -4.30606561 .66298021 .2293244$

H $-0.01876270 .9178372-5.1446224$

H $-0.28655773 .4047773-4.8588396$

H $-0.01769574 .3952711-2.5954516$

H $0.5161783-0.4572018-3.1267546$

C 0.47912534 .25381640 .2155954

C 0.63764934 .60015941 .5474754

C 0.90276933 .56109522 .4364824

C 1.01229132 .23384622 .0436225

H 1.21648731 .47951922 .7908623

H 0.55730835 .62682601 .8743404

H -5.4456535 -2.1426597 -0.3933676

H -7.8383570 - 1.63831770 .0859324

H -6.69061192.1794762 1.7140834

H -8.4642668 0.52809121 .1420503

H $4.1637113-1.87789083 .3593144$

H - $0.0059167-2.30679184 .4087414$

C $2.91949620 .0829822-0.2298566$

N $1.3357973-0.90337281 .6531974$

F $5.88181731 .0749372-2.1990457$

F $5.6976804-1.21098781 .9790104$

F 1.06748333 .88227223 .7779903

F $0.21592235 .3022854-0.6744536$

Complex 1 (triplet; ptpy-localized)

Ir $0.88740180 .0030822-0.0890301$

C $2.6711477-1.06496772 .0049258$

C $3.5625207-0.52688980 .9789049$

C $4.9644770-0.55067581 .0205099$

C $5.7675618-0.01494180 .0266519$

C $5.11174480 .5719492-1.0525612$

C $3.72765370 .6318012-1.1609471$

C 0.81039781 .89600220 .6379109

C $0.54522782 .8921002-0.3543891$

C $0.41022382 .4070593-1.7237641$

C $0.15313583 .1763731-2.8745042$

C $0.06359182 .5620333-4.1207610$

C $0.23061781 .1751843-4.2231332$

C $0.48033180 .4481712-3.0656081$

N $0.56850881 .0419842-1.8497871$

$\mathrm{N}-1.1666952-0.37699080 .0103059$

C $-1.5924552-1.6436828-0.5672581$

C $-0.6179882-2.4911538-1.0857291$ 
C $-0.8677443-3.7752489-1.6602701$

C $0.1742518-4.5461477-2.1138772$

C $1.5136978-4.0378870-1.9975761$

C $1.7243277-2.7794689-1.4431741$

N $0.7229458-1.9842218-0.9985241$

H $6.8452696-0.05049680 .0934529$

H $3.29356071 .1079632-2.0307790$

C $3.0452549-1.68859283 .2093149$

C $2.0693868-2.12882774 .1007160$

C $0.7166248-1.94406573 .7915469$

C $0.3848878-1.32716182 .5907699$

H -0.6398593 -1.1462948 2.3002919

H $2.3618237-2.60691275 .0291070$

H $2.3616227-4.6208228-2.3363322$

H - $0.0027112-5.5225338-2.5497340$

H - $1.8979903-4.1091770-1.7164541$

H $2.7242738-2.3720719-1.3520751$

$\mathrm{N}-2.9468053-1.7522358-0.4856281$

C $-3.3272522-0.58341480 .1160899$

$\mathrm{N}-2.26892620 .27627020 .4280969$

C $-4.6948024-0.23760980 .4135809$

C -5.7356654-1.1343588 0.0800639

C $-7.0585395-0.80516780 .3658289$

C -7.36293320 .41714220 .9851069$

C -6.33487331 .31245821 .3187139$

C -5.00859510 .99249221 .0369049$

H -4.2026873 1.67253821 .2859149

H $0.16722180 .6645242-5.1760093$

H -0.1343993 3.1590172 -5.0042363

H $0.02770674 .2429882-2.7738250$

H $0.6133238-0.6239778-3.0757240$

C 0.43797084 .23029720 .0522179

C 0.58080784 .64247331 .3663989

C 0.84550083 .64643132 .3031260

C 0.96474282 .30264411 .9734179

H 1.16884971 .59026832 .7618140

F $0.17409085 .2347070-0.8903031$

H 0.49268185 .68289341 .6435779

F 0.99714284 .03000323 .6323448

H -5.4761072 -2.0713619-0.3982561

H -7.8552729 -1.4953707 0.1083549

H -6.5724974 2.2571222 1.7966429

H -8.39508590.6706872 1.2058018

H $4.0934118-1.81430783 .4312538$

H - $0.0685182-2.26743284 .4636091$

C $2.92105570 .0818892-0.1483091$

N $1.3312807-0.90280881 .7173129$

F $5.89178891 .1182352-2.0668732$

F $5.6283578-1.14150482 .1048499$

Complex 2 (S0)

Ir -0.92411950 .01899280 .1012967$ 
C - $2.6195654-0.9743323-2.0945203$

C $-3.5655436-0.5234372-1.0701732$

C $-4.9613456-0.6223492-1.2280832$

C $-5.7726215-0.1526282-0.2085503$

C -5.25702130 .40737480 .9563687$

C -3.87124050 .48109081 .0658688$

C $-0.85340551 .9655557-0.4754393$

C -0.56557152 .88869270 .5698718$

C -0.41677952 .29403291 .8959988$

C - 0.13188652 .98903283 .0814498

C - 0.03288352 .30445084 .2890597

C -0.22336550 .91731984 .3038178$

C -0.50000950 .26795283 .1067958$

$\mathrm{N}-0.58903250 .93225681 .9309388$

N $1.1615035-0.35700520 .0239837$

C $1.5627335-1.58590020 .4678937$

C $0.5625285-2.52277510 .9063097$

C $0.8448405-3.82397031 .3495667$

C $-0.2042195-4.65090321 .7417788$

C - $1.5214395-4.16802921 .6848407$

C - $1.7443655-2.86779921 .2379797$

$\mathrm{N}-0.7293545-2.05306930 .8588027$

C $-2.9544455-1.5319682-3.3365292$

C $-1.9517995-1.8850672-4.2373633$

C $-0.6135285-1.6701742-3.8888943$

C $-0.3229965-1.1185263-2.6454503$

H $0.6890325-0.9071452-2.3316583$

H - $2.2093225-2.3119623-5.2004113$

$\mathrm{H}-2.3602085-4.78640501 .9805618$

H - 0.0047685 -5.6597413 2.0864628

H $1.8781074-4.14709511 .3704668$

H - $2.7386085-2.44585531 .1741157$

N $2.9056414-1.73477120 .3962938$

C $3.3148535-0.5287002-0.1150613$

N $2.27748140 .3363018-0.3552882$

C $4.7114244-0.1871812-0.3782742$

C $5.7293125-1.1212322-0.1109383$

C $7.0652905-0.7993242-0.3599002$

C $7.40450630 .4569368-0.8784872$

C $6.39498051 .3905798-1.1466642$

C $5.05752141 .0739548-0.8992792$

H $4.27113971 .7918138-1.1029243$

H -0.15975350.3476888 5.2226496

H 0.18789952 .84106985 .2050648

H 0.01066754 .06124883 .0501729

H $-0.6595465-0.79910323 .0642658$

C -0.44076354 .27225260 .3407158$

C $-0.61007554 .7418166-0.9495223$

C $-0.90157053 .8963497-2.0149153$

C - $1.01473552 .5379627-1.7389793$

H $5.4548846-2.08998820 .2907967$

H $7.8424827-1.5278812-0.1492913$

H $6.65048132 .3664938-1.5482803$

H $8.44361410 .7058628-1.0712323$ 
H -3.9953255 -1.6758832 -3.5963022

H $0.1937895-1.9145922-4.5679364$

C - 2.96705760 .03752680 .0978457

$\mathrm{N}-1.2987405-0.7944272-1.7644293$

$\mathrm{H}-0.21867554 .97613481 .1323997$

H - $1.03519454 .2773877-3.0182332$

H -5.9048676 0.77339181 .7414158

H -5.4201187 -1.0441522-2.1133713

F $-0.49167256 .1089059-1.1946773$

F - $1.31682551 .7233967-2.8405203$

F $-7.1562437-0.2449902-0.3486033$

F-3.3841025 1.0445368 2.2543338

Complex 2 (triplet; ppy-localized)

Ir -0.96355180 .11275930 .0825511$

C $-2.7580378-0.9958187-1.9739318$

C -3.6193897-0.4791927-0.9900759

C $-5.0496928-0.5285497-1.0518189$

C $-5.76816580 .0351413-0.0309449$

C -5.19566790 .67820131 .1008671$

C -3.80953790 .71981131 .1398941$

C $-0.75910792 .0091143-0.5756299$

C -0.33989782 .94520630 .4151811$

C -0.20505682 .40494841 .7680161$

C 0.18611713 .12389432 .9067190

C 0.25596622 .48748034 .1434439

C -0.06981981 .12898834 .2324780$

C -0.44931780 .45516933 .0768581$

$\mathrm{N}-0.51102081 .07343231 .8765021$

N $1.1116152-0.38288680 .0113311$

C $1.4557121-1.62725970 .4565941$

C $0.4158782-2.51832880 .9025241$

C $0.6401222-3.83765471 .3237551$

C $-0.4443298-4.61649381 .7195011$

C $-1.7359719-4.06786981 .6914691$

C $-1.8995858-2.75006571 .2702771$

$\mathrm{N}-0.8490778-1.98528180 .8876741$

C $-3.1355629-1.6500407-3.1875500$

C $-2.1819348-2.0777937-4.0813147$

C $-0.8032958-1.8542417-3.7934570$

C $-0.4565278-1.2134297-2.6110989$

H $0.5751632-1.0004027-2.3650250$

H -2.4725339 -2.5711747 -5.0021927

H -2.6002979 -4.6489049 1.9890161

H $-0.2911188-5.63996282 .0440381$

H $1.6555600-4.21338861 .3229491$

H -2.8706548 -2.2740008 1.2330691

N 2.7912581 -1.8291298 0.4017671

C $3.2568231-0.6385117-0.0994339$

N $2.25970100 .2689723-0.3472539$

C $4.6693833-0.3555778-0.3464119$

C $5.6431730-1.3333278-0.0715339$ 
C $6.9943440-1.0680097-0.3047639$

C $7.39255500 .1742473-0.8149219$

C $6.42696841 .1510382-1.0909759$

C $5.07460340 .8910033-0.8594679$

H $4.32207521 .6419092-1.0716169$

H - 0.03268080 .59997135 .1765512

H 0.55861823 .04069725 .0254372

H 0.43429914 .17352932 .8195971

H $-0.7151898-0.59170973 .0887211$

C -0.09500584 .29440110 .1100061$

C $-0.27432484 .7147101-1.1982589$

C $-0.69726483 .8565563-2.2079429$

C $-0.93158492 .5302562-1.8622898$

H $5.3231581-2.29113190 .3225391$

H $7.7371724-1.8300878-0.0891639$

H $6.72833142 .1158543-1.4874949$

H $8.44331670 .3788333-0.9960559$

H -4.1887707 -1.7967857 -3.3969979

H $-0.0280699-2.1621138-4.4835059$

C -2.95528190.1771443 0.1739851

$\mathrm{N}-1.3775838-0.8063667-1.7104658$

H 0.22552925 .01209440 .8537611

H - $0.84311384 .2048725-3.2212140$

H -5.8120839 1.11298821 .8733311

H -5.5732109 -0.9850018 -1.8812439

F - $0.03465686 .0473331-1.5146609$

F - $1.37473191 .7052683-2.8938779$

F -7.1551120 -0.0099777 -0.0808349

F -3.2496199 1.3646482 2.2530220

Complex 2 (triplet; ptpy-localized)

Ir -0.89740010 .00349070 .0778346$

C $-2.6639421-0.9517364-2.0801725$

C $-3.5774562-0.4653434-1.0439305$

C $-4.9780130-0.5176383-1.1808995$

C $-5.7576570-0.0168173-0.1519844$

C -5.20571140 .53206371 .0012726$

C -3.81700320 .56070971 .0893386$

C $-0.80111211 .9629176-0.4802794$

C -0.50036712 .86792070 .5770955$

C -0.35378512 .25300771 .8956166$

C -0.06720512 .92849473 .0918906$

C 0.03075892 .22325074 .2880094

C -0.16055210 .83592674 .2816928$

C - 0.44108210 .20456773 .0757575

N -0.52948210 .89151261 .9129266$

N $1.1451029-0.3704193-0.0511974$

C $1.5670278-1.67684630 .4319406$

C $0.5869219-2.55702730 .8820675$

C $0.8267849-3.88186951 .3593356$

C $-0.2215831-4.67894051 .7481605$

C - $1.5581751-4.15629641 .6638476$ 
C - $1.7596421-2.85817141 .2072325$

$\mathrm{N}-0.7513091-2.03668230 .8310815$

C $-3.0390811-1.5156173-3.3082935$

C $-2.0660951-1.9149384-4.2212436$

C $-0.7152601-1.7415174-3.8984785$

C $-0.3854311-1.1807573-2.6697166$

H $0.6387879-1.0057843-2.3753144$

H -2.3549801 -2.3486404 -5.1722304

H -2.4105022 -4.7586362 1.9541126

H $-0.0518311-5.68623042 .1104095$

H $1.8549478-4.22496531 .3950806$

H - $2.7563960-2.43844031 .1419086$

N $2.9216688-1.77704530 .3562306$

C $3.3057668-0.5623474-0.1469614$

N $2.25041890 .3174237-0.4001365$

C $4.6762090-0.1927923-0.4008804$

C $5.7148500-1.1120264-0.1277744$

C $7.0403471-0.7599373-0.3714825$

C $7.34946780 .5079246-0.8881704$

C $6.32348491 .4258216-1.1613154$

C $4.99470191 .0832137-0.9208394$

H $4.19025571 .7799147-1.1245595$

H - 0.09654410 .25231875 .1916178

H 0.25070992 .74518075 .2127984

H 0.07450394 .00127063 .0788136

H $-0.6017211-0.86129233 .0080176$

C -0.36152214 .25332670 .3660705$

C $-0.52911014 .7410367-0.9179874$

C $-0.83089213 .9132856-1.9947774$

C $-0.95737212 .5525716-1.7353495$

H $5.4516850-2.08423320 .2718555$

H $7.8354092-1.4676593-0.1605045$

H $6.56465102 .4056466-1.5602295$

H $8.38356880 .7792777-1.0762515$

H -4.0886671 -1.6322803 -3.5451984

H $0.0713889-2.0281963-4.5852086$

C -2.94353910.07988270.1114746

N - $1.3325471-0.8066133-1.7779284$

$\mathrm{H}-0.13075414 .94508681 .1660616$

H -0.9621361 $4.3098214-2.9924435$

H -5.8284273 0.92255661 .7947436

H -5.4637079 -0.9281994 -2.0569474

F - $1.26514011 .7510386-2.8449413$

F $-0.39541116 .1102017-1.1455894$

F -7.1454902 -0.0624643 -0.2711104

F-3.2953820 1.1177397 2.2660325

Complex 3 (S0)

Ir $0.4083046-0.2923741-0.1951362$

C $2.2394366-1.15249121 .9606549$

C $3.1310836-0.69472910 .8668348$

C $4.5520824-0.79830810 .8006628$ 
C $5.2510913-0.2080271-0.2561812$

C $4.56247460 .4533119-1.2749732$

C $3.16922150 .4887969-1.2686652$

C 0.19169861 .61100480 .4400978

C $-0.13264642 .5840927-0.5550022$

C $-0.34643642 .0008959-1.8978342$

C $-0.86331242 .6254288-3.0460091$

C $-0.97632151 .9282959-4.2473490$

C $-0.58341240 .5896778-4.3087170$

C $-0.1287814-0.0145141-3.1437092$

N $-0.02544840 .6583628-1.9718492$

$\mathrm{N}-1.6359304-0.8668861-0.1268952$

C $-1.9247054-2.1254462-0.5737792$

C $-0.8456874-2.9658402-1.0230311$

C $-1.0139374-4.2867860-1.4656192$

C $0.1012146-5.0154051-1.8703471$

C $1.3696836-4.4152301-1.8277231$

C $1.4785796-3.1007510-1.3806891$

N $0.3987536-2.3810862-0.9869331$

H $6.3269766-0.2933631-0.3023342$

H 2.6498617 $0.9616988-2.0932751$

C $2.5961295-1.55273213 .2591209$

C $1.6177046-1.92152014 .1828420$

C $0.2700396-1.87504303 .8199289$

C $-0.0552444-1.43299822 .5424778$

H - $1.0783634-1.32369522 .2120278$

H $1.9113266-2.22632205 .1808426$

H $2.2577256-4.9546622-2.1335242$

H -0.0097504 -6.0379990 -2.2139232

H -2.0138935 -4.7020701 -1.4759392

H $2.4327786-2.5936941-1.3291072$

N -3.2477395 -2.3958641 -0.4984682

C $-3.7649242-1.23388720 .0185588$

$\mathrm{N}-2.8102235-0.27888820 .2589828$

C $-5.1863386-1.02262520 .2860408$

C $-6.1132876-2.04893310 .0267718$

C $-7.4725224-1.85148110 .2796158$

C $-7.9251491-0.62968710 .7938368$

C -7.00639450 .39543691 .0537609$

C -5.64617460 .20333290 .8027018$

$\mathrm{H}-4.93012440 .99295190 .9999868$

H $-0.64749040 .0169789-5.2254522$

H - $1.37517352 .4290019-5.1221139$

H -1.2040194 $3.6438078-3.0098002$

H $0.1514386-1.0570512-3.1167681$

C $-0.19783043 .9596130-0.1804042$

C -0.03575044 .33330411 .1551708$

C 0.21565763 .36719792 .1303518

C 0.35125362 .02983381 .7710569

H 0.57385261 .30510282 .5425019

H - 0.09471745 .37557661 .4322299

H -5.7517253 -2.9899563 -0.3718142

H -8.1792642 -2.6500932 0.0754838

H -7.3503543 1.34525491 .4515659 
H -8.9821550 - 0.47771110 .9894938 H $3.6250297-1.55079213 .5713477$ H - $0.5171135-2.14686914 .5118768$ C $2.4269305-0.1000532-0.2297392$ N $0.8960956-1.07751121 .6441969$ C $5.32438841 .1458228-2.3550262$ C $5.3957084-1.56634411 .7724209$ C $-0.38994345 .0974656-1.1362872$ C 0.45213363 .78641593 .5418949 F - 0.22705554 .95574303 .8710759 F 1.80343664 .03419983 .8026829 F 0.05918262 .80933894 .4549019 F -0.1876744 $6.3385278-0.5472962$ F - $1.68541235 .1561610-1.6829602$ F $0.48527665 .0429598-2.2245111$ F $5.5284308-0.93852323 .0235999$ F $4.8862594-2.84200702 .0322528$ F $6.6948446-1.75975521 .3212408$ F $5.55851972 .4901069-2.0645821$ F $4.64404471 .1244059-3.5718882$ F $6.57377850 .5698999-2.5754361$

Complex 3 (triplet; ppy-localized)

Ir $0.4765482-0.2196400-0.1737765$ C $2.4303952-1.05733701 .8737345$

C $3.2126933-0.53239400 .8164625$

C $4.6629761-0.55367000 .6487745$

C $5.25216140 .2197230-0.3390115$

C $4.49421340 .9812510-1.2594045$

C $3.09124120 .8997060-1.2250695$

C 0.03979521 .61604900 .5156905

C $-0.41508472 .5683410-0.4471395$

C $-0.54398072 .0151870-1.8153755$

C $-1.11609682 .6168530-2.9489874$

C - $1.13724281 .9497370-4.1730527$

C $-0.59735680 .6659310-4.2726626$

C $-0.08350180 .0797030-3.1225435$

$\mathrm{N}-0.06502080 .7255550-1.9334426$

$\mathrm{N}-1.5268038-0.9581930-0.1150445$

C $-1.7111568-2.2460270-0.5393955$

C $-0.5644088-3.0112310-0.9587675$

C $-0.6244798-4.3566108-1.3507845$

C $0.5487022-5.0069089-1.7251385$

C $1.7637932-4.3048478-1.7047095$

C $1.7651483-2.9688280-1.3114075$

N $0.6266482-2.3268979-0.9504315$

H $6.32948530 .2038290-0.4438555$

H $2.51865131 .4171680-1.9851856$

C $2.8622802-1.55046693 .1472195$

C $1.9561113-2.04543904 .0607024$

C $0.5731062-2.06061493 .7519894$

C $0.1607932-1.53577002 .5331064$ 
H $-0.8851028-1.47964902 .2613635$

H $2.3027392-2.40249495 .0238905$

H $2.6940862-4.7821260-1.9867305$

H $0.5231092-6.0480328-2.0272655$

H - $1.5869788-4.8524309-1.3451606$

H $2.6741372-2.3834660-1.2798585$

$\mathrm{N}-3.0138468-2.5985279-0.5177705$

C $-3.6312448-1.4584090-0.0593465$

$\mathrm{N}-2.7538787-0.43522190 .1898105$

C $-5.0717818-1.33978190 .1485075$

C $-5.9155809-2.4343820-0.1175365$

C $-7.2933747-2.32560300 .0811955$

C $-7.8471924-1.12625600 .5474125$

C $-7.0116078-0.03354800 .8138975$

C -5.6334006 - 0.13646300 .6168005

$\mathrm{H}-4.98085470 .70451200 .8215515$

$\mathrm{H}-0.58920080 .1210120-5.2081613$

H - $1.57958192 .4318461-5.0371823$

$\mathrm{H}-1.56828373 .5893349-2.8882026$

H $0.3159512-0.9240430-3.1237406$

C $-0.66848583 .9029050-0.0139985$

C -0.54939984 .23648001 .3375454$

C -0.15929683 .27943612 .2759045$

C 0.15319221 .98837211 .8640055

H 0.47980421 .26271302 .5962685

H - 0.75526785 .24767681 .6573085

H -5.4758379 -3.3574090 - 0.4775625

H $-7.9358079-3.1756800-0.1267565$

$\mathrm{H}-7.43474880 .89837401 .1756955$

H -8.9185397 - 1.04383100 .7018615

H $3.9054202-1.49806193 .4108634$

H - $0.1608848-2.44046694 .4508434$

C $2.42999920 .1350910-0.2626995$

N $1.0319172-1.03910291 .6268485$

C $5.17726841 .8514961-2.2381975$

C $5.5701393-1.41585201 .4465665$

C - $1.03069185 .0426068-0.9186535$

C 0.02228623 .67831703 .7029405

F - 0.85635474 .68951414 .0820833

F 1.30756324 .16215613 .9558955

F - 0.17161382 .61117694 .5743432

F - $1.03212576 .2674188-0.2669855$

F -2.3135087 $4.9189272-1.4806635$

F - $0.14840085 .1779050-1.9929976$

F $5.8120054-0.95369902 .7617004$

F $5.0795754-2.71945411 .6030084$

F $6.8352073-1.54122500 .8779845$

F $5.38147723 .1632161-1.7800955$

F $4.45125521 .9840190-3.4263784$

F $6.44613201 .3765540-2.5826456$

Complex 3 (triplet; ptpy-localized) 
Ir $0.4007242-0.3045132-0.1543294$ C $2.2764232-1.09352021 .9902897$ C $3.1432541-0.62393820 .8873706$ C $4.5663763-0.69303020 .8052037$ C $5.2389093-0.0808412-0.2562214$ C $4.52416620 .5685798-1.2642983$ C $3.12934530 .5668908-1.2445253$ C 0.12473921 .60919280 .4470737 C $-0.21973382 .5548658-0.5685093$ C $-0.37914881 .9450038-1.9095883$ C $-0.85317582 .5422308-3.0909842$ C $-0.91102381 .8210067-4.2822434$ C $-0.50505380 .4850968-4.3039562$ C $-0.0854928-0.0921352-3.1123744$ $\mathrm{N}-0.03529980 .6061788-1.9517634$ $\mathrm{N}-1.6009978-0.9030102-0.0575263$ C $-1.8713829-2.2488463-0.5365053$ C $-0.7999877-3.0144632-0.9909923$ C $-0.9029358-4.3496654-1.4876953$ C $0.2217762-5.0230544-1.8991144$ C $1.4921352-4.3610123-1.8198884$ C $1.5569192-3.0553313-1.3426714$ N $0.4709742-2.3558953-0.9375804$ H $6.3159901-0.1405032-0.3146004$ H $2.59146411 .0274808-2.0641154$ C $2.6586631-1.47183833 .2896136$ C $1.7037263-1.85805514 .2281564$ C $0.3494592-1.85193923 .8802367$ C - $0.0019548-1.42710522 .6054977$ H - $1.0314768-1.35180232 .2871846$ H $2.0172093-2.14632825 .2250036$ H $2.4021202-4.8636611-2.1238242$ H $0.1577762-6.0371683-2.2756974$ H - $1.8888468-4.7996691-1.5216234$ H $2.5045153-2.5343433-1.2802163$ $\mathrm{N}-3.2060737-2.5024002-0.4639334$ C $-3.7290038-1.34035720 .0311107$ $\mathrm{N}-2.7768177-0.34296520 .2858227$ C -5.1314177 - 1.12508720 .2777297 C $-6.0595459-2.15903930 .0128847$ C - $7.4163150-1.95501330 .2498707$ C $-7.8668741-0.72328320 .7511036$ C -6.9518890 0.30768481 .0157027 C -5.59251870 .11365580 .7825336$ H -4.87262960.89892080.9798157 H -0.5278398 -0.1047862 -5.2116572 H - $1.27574972 .3032899-5.1820432$ H -1.1987487 $3.5589897-3.0937063$ H $0.2086572-1.1295492-3.0495395$ C $-0.35254883 .9314487-0.2120884$ C -0.21718984 .32975461 .1195416$ C 0.06787723 .39022592 .1111907 C 0.25648722 .05394781 .7727816 H 0.49786121 .34713482 .5557896 
H -0.3274718 5.37152691 .3817997

H -5.6879229 -3.1001761 -0.3746913

H -8.1266016 -2.7494061 0.0460447

H -7.3032908 1.25852981 .4021196

H -8.9254145 - 0.56770520 .9338046

H $3.6915093-1.43947623 .5872627$

H $-0.4210948-2.14501814 .5821599$

C $2.4152802-0.0479662-0.2033724$

N $0.9261812-1.05166721 .6907396$

C $5.25632251 .2871808-2.3462683$

C $5.4375983-1.44427921 .7643287$

C 0.27256523 .83909483 .5184187

C - $0.60249485 .0477426-1.1795614$

F $5.5813361-0.80936423 .0125397$

F $4.9556242-2.72663312 .0386597$

F $6.7332183-1.61573721 .2931327$

F $4.56607641 .2563658-3.5577635$

F $5.45707902 .6371019-2.0508133$

F $6.51954130 .7485478-2.5821403$

F - $0.51430186 .3039319-0.5946034$

F $0.30429225 .0582578-2.2435273$

F - $1.88278675 .0023958-1.7622943$

F 1.61292824 .11994983 .7981237

F - 0.11645372 .87009574 .4421896

F - 0.43759584 .99927883 .8160165

Complex 4 (S0)

Ir $-0.6065714-0.09972650 .1291570$

C -2.4271034 -0.8231195 -2.0521519

C $-3.2924424-0.2384565-1.0213930$

C $-4.6768004-0.1857355-1.2055990$

C $-5.48675360 .4404315-0.2606180$

C -4.90029971 .01809650 .8626240$

C -3.51149950.9609215 1.0528940

C $0.03774461 .8340516-0.1741530$

C 0.77481152 .32237150 .9573290

C 0.78108251 .48378152 .1554689

C 1.44862461 .77102853 .3565891

C 1.34620660 .90276054 .4387369

C $0.5663775-0.25307554 .3144340$

C - $0.0719864-0.50032553 .1065399$

N 0.03544260 .34250552 .0511780

N $1.3313536-0.9227915-0.0299280$

C $1.4415985-2.25524060 .2465030$

C $0.2534416-2.99220460 .5878170$

C $0.2409746-4.36656830 .8694340$

C - $0.9638784-4.98265051 .1963360$

C $-2.1399175-4.21733061 .2369259$

C - 2.0705994 -2.8568966 0.9465680

$\mathrm{N}-0.9017054-2.24704450 .6287180$

C $-2.8524595-1.4129415-3.2514739$

C $-1.9148395-1.8888804-4.1657200$ 
C $-0.5517664-1.7631194-3.8762691$

C $-0.1722324-1.1772655-2.6742139$

H $0.8637376-1.0374405-2.4052050$

H - 2.2420294 -2.3429105 -5.0944128

H -3.0935343 -4.6641766 1.4899430

H -0.9924635 -6.0437514 1.4182720

H $1.1771796-4.90867870 .8242300$

H - $2.9483844-2.22576040 .9655620$

N $2.7191626-2.68583240 .1432280$

C $3.3956195-1.5444615-0.2106720$

N $2.5790125-0.4482255-0.3263850$

C $4.8386577-1.4882585-0.4357490$

C $5.6213044-2.6479794-0.2867560$

C $7.0011858-2.5983655-0.4959470$

C $7.6179608-1.3935125-0.8565820$

C $6.8430656-0.2361505-1.0070570$

C $5.4629444-0.2799785-0.7986870$

H $4.85773930 .6121375-0.9127550$

H $0.4519485-0.94960355 .1354208$

H 1.86400451 .12263155 .3655200

H 2.04937452 .66631163 .4398689

H -0.6899044 -1.3721835 2.9629750

C 1.43189363 .55524140 .9590230

C $1.36521054 .3865934-0.1545130$

C $0.62348063 .9796136-1.2589290$

C $-0.03583942 .7410186-1.2682851$

H $5.1334018-3.5745994-0.0066480$

H $7.5960435-3.4989025-0.3773800$

H $7.31444440 .7010145-1.2864580$

H $8.6908158-1.3566075-1.0184570$

H -3.9087244 -1.4942605 -3.4708221

H $0.2076916-2.1031775-4.5688882$

C -2.6372644 0.3140095 0.1352940

$\mathrm{N}-1.0895294-0.7318665-1.7808460$

H 1.98240053 .89254241 .8278470

H $0.53915954 .6353706-2.1142321$

H -5.5207106 1.5264935 1.5879110

H -5.1382896 -0.6030855 -2.0920820

C $2.12334055 .6649445-0.1891140$

C $-0.78344142 .4536045-2.5296719$

C $-6.96555530 .4240655-0.4138410$

C -3.02077231 .58800252 .3179300$

F 2.30323456 .20537451 .0851440

F $3.40719365 .5198224-0.7258360$

F $1.49328966 .6356004-0.9642820$

F -2.0810574 $1.9866635-2.3205610$

F - $0.92884943 .5808585-3.3411200$

F - $0.15114741 .5037595-3.3450260$

F $-7.5506755-0.71056550 .1649150$

F $-7.35740240 .4092255-1.7523670$

F -7.5774785 1.5192645 0.1867710

F -4.00314252 .33653352 .9676981$

F - 2.60872040 .63185253 .2628400

F - 1.94638352 .45520862 .1434419 

Complex 4 (triplet; ppy-localized)
Ir $-0.6243119-0.03609580 .1143359$
C - $2.4689290-0.7455949-2.0502812$
C $-3.2826130-0.1991088-1.0588131$
C $-4.7256429-0.2070418-1.1633852$
C $-5.47460200 .4794942-0.2510231$
C -4.85929511 .19960220 .8164029$
C -3.4587068 1.16157820.9811989
C $0.12411511 .8509881-0.1911871$
C 0.89579912 .29120220 .9365639
C 0.82257401 .47590812 .1495229
C 1.48242501 .73804113 .3597799
C 1.29089310 .89989824 .4545220
C $0.4310020-0.19788184 .3332127$
C $-0.1962809-0.42306583 .1146158$
$\mathrm{N}-0.00097790 .38946722 .0495590$
N $1.2865441-0.9462009-0.0119511$
C $1.3292021-2.28656990 .2452849$
C $0.1053990-2.97700880 .5621879$
C $0.0336831-4.35447170 .8202329$
C - $1.1992579-4.92509891 .1240439$
C - $2.3429860-4.11254691 .1699409$
C $-2.2138619-2.75088690 .9069459$
$\mathrm{N}-1.0177690-2.18734980 .6077729$
C -2.8980911 -1.3301099-3.2923640
C $-1.9774179-1.7993598-4.1982709$
C $-0.5898589-1.6949449-3.9013170$
C $-0.1928150-1.1175329-2.6923622$
H $0.8524380-1.0029178-2.4419922$
H $-2.3013630-2.2329178-5.1374933$
H -3.3165619 -4.5240889 1.4060799
H - $1.2742259-5.98808191 .3251709$
Н $0.9469991-4.93413880 .7747039$
H -3.0628049 -2.0813449 0.9384929
N 2.5882550 -2.7716489 0.1680659
C $3.3249040-1.6568577-0.1503701$
N $2.5623720-0.5234678-0.2669871$
C $4.7734359-1.6645458-0.3440081$
C $5.4977319-2.8619518-0.1978111$
C $6.8822983-2.8742898-0.3795231$
C $7.5618251-1.6946728-0.7094391$
C $6.8452389-0.4999168-0.8567991$
C $5.4607432-0.4817758-0.6758921$
H $4.90037710 .4392582-0.7892901$
H $0.2464851-0.86620085 .1647571$
H 1.80129321 .10000325 .3897828
H 2.14269122 .59046823 .4425238
H $-0.8709079-1.25228482 .9686600$
C 1.65008003 .46689910 .9183119
C $1.64289004 .2838402-0.2078681$
C $0.85701413 .9300182-1.3012431$ 
C $0.09978712 .7497232-1.2916921$

H $4.9615281-3.76878170 .0576039$

H $7.4318311-3.8035078-0.2639211$

H $7.36544400 .4177962-1.1131011$

H $8.6381781-1.7060719-0.8504051$

H -3.9566149 -1.3803458 -3.5138181

H $0.1623811-2.0399828-4.5987312$

C -2.60385390.4408462 0.1229579

$\mathrm{N}-1.0764540-0.6631048-1.7885051$

H 2.2322581 3.77039221 .7788689

H $0.81472514 .5843892-2.1608151$

H -5.4729779 1.76773621 .4999859

H -5.2136781 -0.7369318 -1.9701481

C $2.50720915 .4938330-0.2664341$

C $-0.69130592 .5147741-2.5397900$

C $-6.96371390 .4614552-0.3176621$

C -2.9546079 1.89042422 .1850909

F 2.74553216 .03121800 .9987628

F $3.76619225 .2289481-0.8138851$

F $1.95308126 .5040550-1.0476391$

F - $1.99077202 .0763072-2.3069111$

F - $0.82559903 .6736171-3.3087140$

F - $0.10022691 .5784372-3.3982252$

F - - 7.5221350 -0.2619328 0.7413709

F -7.4463532 - $0.1142178-1.4883791$

F $-7.50851411 .7408482-0.2337031$

F -3.9051440 2.75323132.7306100

F -2.6044900 1.0238322 3.2378029

F-1.83113392.6783202 1.9464250

Complex 4 (triplet; ptpy-localized)

Ir $-0.5893410-0.13352130 .1044088$

C $-2.4519660-0.8515143-2.0464021$

C $-3.2954790-0.2421643-1.0137192$

C $-4.6792791-0.1589753-1.1919322$

C $-5.47191500 .4798867-0.2417772$

C -4.86866691 .03583970 .8832017$

C -3.48051400 .95024271 .0665518$

C $0.04510401 .8165597-0.1951162$

C 0.76162602 .31493760 .9452428

C 0.77890901 .46878972 .1394997

C 1.43009301 .76503973 .3473607

C 1.34850000 .88277774 .4204527

C $0.6092841-0.29737534 .2797438$

C - $0.0172250-0.55293233 .0668918$

N 0.06741300 .30726372 .0232879

N $1.3302601-0.9122504-0.0908142$

C $1.4571700-2.32434720 .2244078$

C $0.3088680-3.02424740 .5799977$

C $0.2668400-4.41570040 .9028888$

C $-0.9218390-5.01289741 .2420928$

C - $2.1150890-4.21355151 .2654187$ 
C $-2.0432690-2.86044740 .9520168$

$\mathrm{N}-0.8895250-2.23314640 .6191878$

C $-2.9039290-1.4547833-3.2297932$

C $-1.9889140-1.9644224-4.1475662$

C $-0.6200620-1.8594954-3.8771931$

C $-0.2158530-1.2590394-2.6912991$

H $0.8254340-1.1354023-2.4380643$

H - $2.3369959-2.4309113-5.0623383$

H -3.0717521 -4.6491065 1.5267298

H -0.9665570 -6.0670661 1.4889297

H $1.1996780-4.96737430 .8675727$

H -2.9307790 -2.2406044 0.9717008

N $2.7622719-2.69999930 .1224038$

C $3.4034670-1.5462243-0.2300462$

N $2.5588501-0.4343043-0.3650932$

C $4.8239191-1.4414004-0.4430702$

C $5.6461861-2.5807433-0.2807762$

C $7.0204022-2.4821944-0.4814492$

C $7.5932505-1.2529174-0.8453232$

C $6.7835682-0.1181163-1.0087942$

C $5.4078052-0.2058523-0.8099812$

H $4.76807000 .6601367-0.9310032$

H $0.5154390-1.00700645 .0920139$

H 1.85176111 .11115775 .3533159

H 1.99941212 .67936163 .4443968

H -0.6017710 -1.4440143 2.9037498

C 1.39414713 .56074670 .9582778

C $1.32657404 .3932877-0.1548792$

C $0.61242203 .9725817-1.2726663$

C $-0.02108802 .7210577-1.2911052$

H $5.1811359-3.51775140 .0017798$

H $7.6497309-3.3569593-0.3555352$

H 7.2290490 0.8301707 -1.2900572

H $8.6650632-1.1799733-1.0002042$

H -3.9644360 - $1.5244703-3.4311372$

H $0.1240870-2.2298083-4.5709242$

C - 2.62266490 .29504970 .1386428

$\mathrm{N}-1.1104380-0.7776054-1.7947972$

H -5.4754102 1.54997661 .6157667

H -5.1529281 -0.5630493 - 2.0779323

H $0.53216904 .6252017-2.1308393$

H 1.92610003 .90761981 .8350298

C $-0.73249702 .4060636-2.5663573$

C $2.06339415 .6837907-0.1770752$

C $-6.95106190 .5012307-0.3907022$

C - 2.97522901 .55940872 .3343748

F - - $.5658471-0.61427530 .1927918$

F -7.3466131 $0.4939597-1.7284443$

F-7.5318448 1.6150717 0.2078668

F - 1.90867002 .43755562 .1555699

F -3.9504969 2.2942996 3.0103227

F - 2.54262590 .59428273 .2584167

F $3.35989505 .5581298-0.6915523$

F 2.2143440 6.22809081 .0991687 
F $1.43338716 .6441899-0.9643042$

F - $0.07512401 .4361067-3.3373603$

F -2.0359959 $1.9448637-2.3845271$

F - $0.85138803 .5132118-3.4095203$

Complex 5 (S0)

Ir -0.90831980 .05046240 .0891958$

C $-2.5726787-1.4278086-1.8315972$

C $-3.5251209-0.7833427-0.9280052$

C $-4.9208769-0.9387776-1.0342382$

C $-5.7671198-0.2805736-0.1437392$

C -5.21659890 .53827330 .8546568$

C -3.83008090.6940004 0.9631448

C $-0.87810581 .7842504-0.9553692$

C $-0.67683282 .9511893-0.1637062$

C -0.55085782 .71746831 .2689188$

C -0.33485783 .69716142 .2546807$

C -0.23846583 .33999443 .5954008$

C -0.35951281 .99005843 .9548028$

C - 0.56907781 .04997242 .9532457

$\mathrm{N}-0.66513781 .39522541 .6451538$

N $1.1994993-0.32499060 .1354318$

C $1.5943252-1.41238160 .8645558$

C $0.5938792-2.22237371 .5085388$

C $0.8812822-3.37552262 .2560208$

C $-0.1660128-4.09292482 .8275447$

C - $1.4860607-3.65079262 .6437457$

C - $1.7132728-2.49941971 .8930428$

$\mathrm{N}-0.6999438-1.79148771 .3364988$

H -6.8429030 -0.3974746 -0.2258662

H -3.4323677 1.33918441 .7405428

C $-2.8877248-2.2536077-2.9240412$

C $-1.8755878-2.7772057-3.7247111$

C $-0.5400238-2.4671967-3.4339912$

C $-0.2639638-1.6510576-2.3420882$

H $0.7431192-1.3633646-2.0742001$

H - $2.1218128-3.4128397-4.5684621$

H - $2.3234788-4.18757993 .0730899$

H $0.0369522-4.98641283 .4082089$

H $1.9166533-3.67393662 .3632458$

H - 2.7093807 -2.1132047 1.7196408

N $2.9378952-1.57954560 .8391268$

C $3.3544742-0.53574870 .0528048$

N $2.32253020 .2490694-0.3952112$

C $4.7530462-0.2739616-0.2817162$

C $5.7672874-1.12104060 .2020148$

C $7.1044990-0.8749296-0.1174282$

C $7.44916120 .2179974-0.9230802$

C $6.44343441 .0648663-1.4070142$

C $5.10487620 .8234574-1.0904102$

H $4.32076321 .4747573-1.4594702$

H - 0.28812481 .67065334 .9872320 
H - 0.06871284 .09831454 .3520387

H - 0.23943384 .73367861 .9580198

H $-0.6645188-0.00344363 .1733399$

C $-0.60701584 .2294796-0.7515102$

C $-0.74095784 .3724725-2.1297552$

C $-0.94733083 .2333853-2.9237923$

C - $1.01629581 .9629984-2.3441863$

H - $1.17486981 .1029344-2.9858512$

H $-0.68697185 .3561232-2.5848152$

H $5.4883381-1.96327070 .8249838$

H $7.8786160-1.53539470 .2620068$

H $6.70292631 .9146214-2.0314011$

H $8.48924510 .4079134-1.1705322$

H -3.9249748 -2.4734037 -3.1426992

H $0.2736292-2.8452237-4.0404884$

C -2.94367680 .03991540 .0841698$

N -1.2502208 -1.1528736 -1.5547682

$\mathrm{H}-5.87381491 .05896741 .5460538$

H -5.3513249 -1.5679857 -1.8083132

H - $1.05435083 .3401663-3.9999072$

H - $0.44765985 .1120865-0.1387322$

Complex 5 (triplet; ppy-localized)

Ir $0.93317780 .1117885-0.0670439$

C $2.7427668-1.33612651 .7450751$

C $3.5910799-0.67581250 .8293350$

C $5.0217990-0.73449950 .8436050$

C $5.7555546-0.0522165-0.1060670$

C $5.12175360 .7333375-1.1157099$

C $3.72870370 .8191205-1.1433589$

C 0.80586381 .80880860 .9972301

C 0.52092382 .97600750 .2283781

C $0.37573882 .7643776-1.2087569$

C $0.06713783 .7455804-2.1661838$

C - $0.06265323 .3999405-3.5082530$

C $0.11592382 .0641215-3.8939520$

C $0.42214181 .1231786-2.9169589$

N $0.55599881 .4604915-1.6123270$

$\mathrm{N}-1.1653492-0.3749135-0.1011520$

C $-1.5257062-1.4968795-0.7916690$

C $-0.5022732-2.2930346-1.4186490$

C $-0.7532122-3.4922435-2.1034600$

C $0.3154688-4.1907175-2.6594650$

C $1.6173407-3.6832035-2.5252169$

C $1.8062188-2.4849055-1.8397100$

N $0.7714458-1.7976714-1.3004429$

H $6.8404159-0.1117735-0.0861479$

H $3.25320691 .4319196-1.9035289$

C $3.1291439-2.17236352 .8347050$

C $2.1885528-2.75287063 .6538860$

C $0.7994138-2.50327043 .4072880$

C $0.4368258-1.68549852 .3485950$ 
H - $0.5994882-1.45496442 .1374100$ H $2.4898888-3.38673744 .4803098$ H $2.4699888-4.2055523-2.9419719$ H $0.1424138-5.1212843-3.1893080$ H - $1.7756822-3.8413935-2.1742519$ H $2.7854747-2.0426564-1.7087929$ $\mathrm{N}-2.8640292-1.6940634-0.7709289$ C $-3.3138412-0.6299164-0.0300769$ $\mathrm{N}-2.30623010 .19671650 .3939551$ C $-4.7215582-0.39066050 .2826160$ C $-5.7103010-1.2755075-0.1857719$

C - $7.0563603-1.05071640 .1114670$

C -7.43499520.05797350.8793300

C -6.45455220 .94198761 .3484491$

C -5.10735140.7220095 1.0539191

$\mathrm{H}-4.34264711 .40094551 .4137011$ H $0.01813081 .7562095-4.9275528$ H - $0.30394424 .1576188-4.2456628$ $\mathrm{H}-0.07606524 .7708623-1.8503790$ H $0.57008680 .0790965-3.1544319$ C 0.39022884 .23119660 .8478401 C 0.53470284 .34538772 .2300810 C 0.81223383 .20385962 .9989871 C 0.94657481 .95345352 .3901340 H 1.15768181 .08341553 .0003761 H 0.43110585 .31391072 .7082390 H -5.4051223 -2.1302445 -0.7786080 H -7.8106952 -1.7404934 -0.2552529 H -6.74048531 .80314051 .9449991$ $\mathrm{H}-8.48182300 .23087851 .1100831$ H $4.1858758-2.34168943 .0099150$ Н $0.0319978-2.93669064 .0364569$ C $2.92410080 .1438435-0.2048339$ N $1.3490018-1.11013651 .5257491$ H $5.72501461 .2643086-1.8444580$ H $5.5351290-1.31765541 .6013530$ H 0.92306583 .29367074 .0755399 Н 0.17507185 .11920480 .2613271

Complex 5 (triplet; ptpy-localized)

Ir -0.87904270 .03377890 .0710004$

C $-2.6331547-1.3597421-1.8357526$

C $-3.5454097-0.6765860-0.9205966$

C $-4.9479015-0.7625611-1.0170636$

C $-5.7538918-0.0692081-0.1164196$

C -5.15648780 .71623890 .8816854$

C -3.76349980 .80381690 .9798664$

C $-0.80428171 .7980619-0.9363756$

C $-0.57354472 .9370909-0.1121596$

C - 0.45554972 .66062701 .3146074

C -0.22604373 .60742892 .3294602$

C -0.13881073 .20786013 .6588753$ 
C -0.28262571 .84987993 .9796644$

C -0.50783470 .94182192 .9524504$

$\mathrm{N}-0.59363271 .33143991 .6559113$

N $1.1738633-0.36771810 .0565254$

C $1.5826953-1.53551200 .8253134$

C $0.5970753-2.28772011 .4603834$

C 0.8335543 -3.4660451 2.2328135

C $-0.2181117-4.15402722 .7865942$

C $-1.5547656-3.66864412 .5715962$

C - $1.7516326-2.51173611 .8246313$

$\mathrm{N}-0.7398437-1.79723211 .2784704$

H -6.8346499-0.1337281 -0.1900576

H -3.3297597 1.42252591 .7593033

C $-2.9993957-2.1753830-2.9201735$

C $-2.0237216-2.7497711-3.7301606$

C $-0.6715277-2.5015342-3.4567095$

C $-0.3454957-1.6928751-2.3739466$

H $0.6770453-1.4554680-2.1175786$

H - 2.3097938 -3.3791210 -4.5659489

H -2.4095576 -4.1903912 2.9851395

H $-0.0510867-5.04961703 .3741194$

H $1.8615853-3.78894012 .3559783$

H -2.7476617 -2.1201331 1.6534014

N $2.9363894-1.66254110 .7820384$

C $3.3333823-0.59719910 .0152154$

N $2.29106320 .2092939-0.4354376$

C $4.7098142-0.3127361-0.3140566$

C $5.7365462-1.16051200 .1588494$

C $7.0663645-0.8920451-0.1595386$

C $7.39154830 .2207989-0.9499886$

C $6.37693421 .0676430-1.4220096$

C $5.04428740 .8072850-1.1089636$

H $4.24826831 .4511510-1.4639627$

H - 0.21890271 .50043495 .0027195

H 0.04009833 .94089304 .4381352

H -0.11517974 .65108382 .0648085$

H $-0.6232097-0.11730713 .1332234$

C $-0.47224674 .2295898-0.6634546$

C $-0.60233274 .4139178-2.0373937$

C $-0.83652473 .3031150-2.8635737$

C $-0.93728072 .0190929-2.3193835$

H - $1.11681471 .1799399-2.9838146$

H - $0.52469975 .4084709-2.4646286$

H $5.4611341-2.01307410 .7683584$

H $7.8520762-1.54560500 .2056894$

H $6.62966221 .9285490-2.0325097$

H $8.42862050 .4272079-1.1959206$

H -4.0482996 -2.3509440 -3.1219126

H $0.1163593-2.9239430-4.0678886$

C - 2.91865260 .11027090 .0914924

$\mathrm{N}-1.2965186-1.1419321-1.5791927$

$\mathrm{H}-5.78195171 .26319591 .5821054$

H -5.4144319-1.3653350 -1.7911227

H $-0.94070073 .4434310-3.9362008$ 


\section{Complex 6 (S0)}

Ir $0.91857770 .0137841-0.0782838$

C $2.5086007-0.58833092 .3306133$

C $3.4878097-0.31780991 .2780902$

C $4.8815615-0.37156391 .4262222$

C $5.7705448-0.08463590 .4024132$

C $5.21321750 .2750811-0.8220148$

C $3.84261150 .3473131-1.0423938$

C 0.82423262 .00839510 .2268632

C $0.63747962 .7779930-0.9636308$

C $0.58048862 .0212921-2.2081088$

C $0.39950462 .5379310-3.5051938$

C $0.37697061 .6825081-4.6029967$

C $0.53710060 .3049351-4.4087466$

C $0.7098956-0.1674069-3.1136037$

N $0.73195360 .6594091-2.0389148$

$\mathrm{N}-1.1754994-0.3840549-0.0577468$

C $-1.5429944-1.6714639-0.3344398$

C $-0.5161244-2.6509089-0.5864958$

C $-0.7722814-4.0027697-0.8556538$

C $0.2997126-4.8660948-1.0702338$

C $1.6094286-4.3655150-1.0110908$

C $1.8042026-3.0126929-0.7410988$

N $0.7672936-2.1640320-0.5348068$

H $6.8390596-0.13424990 .5543992$

H $3.48669860 .6408821-2.0217308$

C $2.7737186-0.92317893 .6706723$

C $1.7211076-1.10346394 .5663444$

C $0.4020376-0.94301394 .1261331$

C $0.1776576-0.62018392 .7921592$

H $-0.8156504-0.46296692 .3960861$

H $1.9293087-1.35670995 .6000210$

H $2.4649765-5.0103628-1.1710138$

H $0.1223796-5.9152419-1.2795748$

H - $1.8018004-4.3370130-0.8871008$

H $2.7921076-2.5750479-0.6872918$

N $-2.8844323-1.8359089-0.2918048$

C $-3.3192325-0.57851090 .0311242$

$\mathrm{N}-2.31147340 .33558010 .1832382$

C $-4.7273085-0.22747190 .2101802$

C $-5.7153013-0.6596249-0.6864318$

C $-7.0579984-0.3330169-0.5210908$

C -7.45306420.45105010.5575932

C -6.49898360 .89757711 .4655772$

C -5.16169430 .55457711 .2903012$

H $0.5253176-0.3906059-5.2387370$

H $0.23540662 .0853521-5.5997390$

H $0.27807563 .6022051-3.6320008$

H $0.8349236-1.2201429-2.9062778$

C $0.52382464 .1715660-0.8472158$ 
C 0.59005464 .84477590 .3605302

C 0.78094764 .06238611 .4965062

C 0.90107262 .67935711 .4579722

H 1.04368072 .14538312 .3876091

F $0.33657464 .9597543-1.9906489$

H 0.49827165 .91988300 .4123192

F 0.85798474 .70910192 .7249542

H 3.7978294 -1.0269499 3.9936391

H -0.4389204 -1.0577529 4.7985393

C 2.94791360 .05093610 .0018922

N $1.1991056-0.46036591 .9140393$

F $6.08046360 .5682771-1.8690338$

F $5.4460714-0.73672992 .6559383$

F -5.3727462 -1.4060809-1.7915078

F -7.9956543 -0.7681149-1.4299308

F -8.7764928 0.78102610 .7255642

F -6.8853586 1.65954122 .5443953

F-4.2647455 0.98213612 .2446543

Complex 6 (triplet; ppy-localized)

Ir $0.93027240 .0467413-0.0753686$

C $2.6266693-0.65878172 .2407264$

C $3.5231845-0.35489371 .2074875$

C $4.9582376-0.39354171 .2766705$

C $5.7721762-0.07420470 .2252694$

C $5.16519160 .3206493-0.9920796$

C $3.78681650 .3922873-1.1534606$

C 0.81373142 .01812230 .3077274

C $0.58775732 .8319632-0.8464936$

C $0.49912832 .1283913-2.1219045$

C $0.26552842 .6973673-3.3874537$

C $0.19911931 .8845573-4.5163454$

C $0.36605830 .5006993-4.3839177$

C $0.5949374-0.0224387-3.1165315$

N $0.66408840 .7635793-2.0154625$

$\mathrm{N}-1.1670926-0.3859887-0.0347606$

C $-1.5246096-1.6752677-0.3131296$

C $-0.4945746-2.6465088-0.5882316$

C - $0.7467497-4.0004986-0.8503426$

C $0.3273634-4.8543309-1.0919325$

C $1.6339424-4.3429459-1.0693016$

C $1.8235654-2.9875568-0.8083526$

N $0.7845893-2.1508397-0.5741136$

H $6.8487024-0.11723970 .3216354$

H $3.38971040 .7162573-2.1074165$

C $2.9239623-1.06342873 .5885114$

C $1.9191393-1.29271974 .4934703$

C $0.5544783-1.11593174 .0894505$

C $0.2741414-0.72937372 .7839285$

H $-0.7421316-0.56604272 .4501215$

H 2.1502563 -1.5942157 5.5083186

H $2.4901804-4.9811829-1.2503166$ 
Н $0.1537003-5.9054369-1.2944146$

H - $1.7736337-4.3439916-0.8547036$

H $2.8074004-2.5373937-0.7876705$

$\mathrm{N}-2.8642026-1.8478018-0.2715106$

$\mathrm{C}-3.3090146-0.59297270 .0501614$

$\mathrm{N}-2.30757860 .32738130 .2031554$

C $-4.7204614-0.25263370 .2221314$

C -5.7043858 -0.7237147 -0.6599116

C -7.0510326 -0.4102967 -0.5019185

C -7.45528280 .40012230 .5535184$

C -6.50576260 .88670131 .4454425$

C -5.16398060 .55675831 .2785784$

H $0.3190284-0.1608687-5.2398986$

H $0.01727442 .3260533-5.4898466$

H $0.13689533 .7653724-3.4670607$

H $0.7307324-1.0821197-2.9542876$

C $0.46521334 .2168023-0.6657466$

C 0.55075834 .83485710 .5710344

C 0.77264044 .00793331 .6696785

C 0.90767142 .62954031 .5680515

H 1.07454842 .05016232 .4654864

H 0.44829135 .90566550 .6721094

H $3.9610862-1.17342773 .8697714$

H - $0.2603866-1.27023474 .7855154$

C $2.93334020 .0628233-0.0922266$

N $1.2401324-0.51408071 .8634554$

F -4.2727166 1.0278663 2.2167744

F -6.9005047 1.67697132.5004114

F -8.7826501 0.71733430 .7137705

F -7.9836546 -0.8844197 -1.3962935

F -5.3549138 -1.4969597-1.7443006

F $6.00711340 .6377053-2.0538496$

F $5.5736884-0.78208772 .4747464$

F $0.24650645 .0527774-1.7672166$

F 0.86637044 .60292932 .9206613

Complex 6 (triplet; ptpy-localized)

Ir $-0.8998513-0.00483460 .0469497$

C - $2.6284552-0.4785216-2.2969074$

C -3.5451012 -0.2052126-1.1910414

C $-4.9455431-0.1978056-1.2736284$

C $-5.77209740 .0918234-0.1998943$

C -5.14353920 .38988641 .0063567$

C -3.7622793 0.40043341 .1622966

C $-0.77586322 .0086324-0.1729613$

C - 0.53108132 .71413931 .0464817

C -0.44570731 .89568042 .2505406$

C -0.21673732 .34433043 .5654968$

C -0.17055331 .43350944 .6171888$

C -0.35332430 .06849944 .3607968$

C $-0.5752883-0.33799463 .0510917$

N -0.6218983 0.5453854 2.0227876 
N $1.1512967-0.4014936-0.0857023$

C $1.5293667-1.77092260 .1784086$

C $0.5339527-2.69970270 .4359937$

C $0.7489627-4.09530850 .6882897$

C $-0.3179823-4.93426580 .8753287$

C - $1.6497033-4.38737080 .8130387$

C - $1.8284083-3.02233450 .5850586$

$\mathrm{N}-0.8066162-2.15848370 .4144647$

H -6.8477262 $0.0895124-0.3012504$

H -3.3489953 0.6458314 2.1322497

C $-2.9718423-0.7724746-3.6290514$

C $-1.9730564-0.9730856-4.5796673$

C $-0.6284953-0.8750437-4.2022273$

C $-0.3272653-0.5896946-2.8755063$

H $0.6892057-0.4854866-2.5246994$

H - 2.2415562 -1.1964167 -5.6062485

H -2.5170382 -5.0222127 0.9472606

H -0.1724963 -5.9910094 1.0648196

H $1.7742878-4.44701880 .7167207$

H -2.8220592 -2.5915765 0.5490856

N $2.8943738-1.89057150 .1330297$

C $3.3056616-0.6262797-0.1469193$

N $2.27532170 .3099034-0.2844833$

C $4.6996476-0.2370416-0.2827904$

C $5.7249538-0.89567970 .4252536$

C $7.0600259-0.52402060 .3066247$

C $7.42066690 .5246884-0.5329303$

C $6.43592661 .1948283-1.2516953$

C $5.10396360 .8158523-1.1273864$

H - $0.3232233-0.66715065 .1548455$

H 0.00634071 .78425345 .6279448

$\mathrm{H}-0.07827133 .39941253 .7419987$

H - $0.7201353-1.37607072 .7889076$

C -0.39568134 .10981140 .9951477$

C $-0.49335934 .8429783-0.1750423$

C $-0.74080834 .1221264-1.3405424$

C $-0.88559432 .7408233-1.3654334$

H - $1.07237132 .2553144-2.3140632$

F - 0.15060834 .83670552 .1678665

H $-0.38294035 .9176105-0.1773593$

F - $0.84969134 .8309405-2.5315313$

H $-4.0139211-0.8324626-3.9014143$

H $0.1732167-1.0119247-4.9170672$

C - 2.9333404 0.09978740.0674737

$\mathrm{N}-1.2963473-0.4061157-1.9451534$

F -5.9470742 0.68560442 .1017748

F -5.5814121 -0.4980536 -2.4854504

F $4.18975471 .4863743-1.9042434$

F $6.78758592 .2189174-2.0988654$

F $8.73622050 .8944434-0.6521643$

F $8.0269668-1.18207571 .0295437$

F $5.4376408-1.91232171 .3022047$ 


\section{Complex 7 (S0)}

Ir $0.07566620 .0687737-0.0353406$

C $2.5347973-0.70959831 .3927983$

C $2.9915021-0.07256330 .1578414$

C $4.32938820 .0910637-0.2298716$

C $4.71351010 .7180547-1.4046596$

C $3.68862621 .2004927-2.2149367$

C $2.34203921 .0737287-1.8955637$

C -0.04957281 .91949170 .7636894$

C $-0.75200282 .8650917-0.0476896$

C - $1.23690792 .3663277-1.3285327$

C - $1.95626583 .0874986-2.3002677$

C $-2.34648882 .4690377-3.4846837$

C - $2.01874671 .1250717-3.7024946$

C - $1.31062180 .4461007-2.7185437$

$\mathrm{N}-0.92521181 .0423227-1.5634937$

$\mathrm{N}-1.7987888-0.66224130 .6561563$

C $-2.1457158-1.91333330 .2369494$

C $-1.2294388-2.6448863-0.5958336$

C $-1.4597558-3.9494775-1.0602177$

C $-0.4982648-4.5680035-1.8542406$

C $0.6816492-3.8767432-2.1740617$

C $0.8581842-2.5841373-1.6869436$

N $-0.0740718-1.9715353-0.9159776$

H $1.60376621 .4818517-2.5742886$

C $3.3352283-1.24946032 .4157854$

C $2.7410112-1.79124443 .5542024$

C $1.3463222-1.79030523 .6747573$

C $0.5866672-1.25366142 .6407464$

H $-0.4926778-1.20769632 .6767354$

H $3.3612443-2.20279634 .3429203$

H $1.4502911-4.3303283-2.7879707$

H -0.6591328 -5.5759424 -2.2211546

H - $2.3853768-4.4372624-0.7813066$

H $1.7471242-2.0065874-1.9041557$

$\mathrm{N}-3.3555087-2.29423630 .7179643$

C $-3.7368018-1.20612531 .4579343$

N $-2.8184339-0.19135331 .4489924$

H -2.3062989 $0.6106757-4.6111426$

H -2.9014918 3.0284006 -4.2297115

H -2.1996099 4.1209878 -2.1100835

H - $1.0348598-0.5922203-2.8308177$

C -0.92298384 .16897670 .4406854$

C - 0.44195884 .59454271 .6670393

C 0.24316023 .64848372 .4252584

C 0.45066722 .34096162 .0069073

H 0.99026321 .66771772 .6592083

H - 0.59317885 .60715882 .0115314

H $4.4085713-1.22898642 .3090682$

H $0.8523532-2.19108834 .5511442$

C $1.96084220 .4330937-0.7019217$

N $1.1614462-0.73644631 .5265733$

F -1.6093859 $5.1227337-0.3243576$ 
F 0.74301424 .04598373 .6616114

F $5.3638893-0.39630130 .5816114$

H $5.75507030 .8277507-1.6698527$

F $4.04391111 .8342177-3.4017617$

C -5.0236019-1.1107313 2.2022224

H $-5.0687488-0.16453032 .7465513$

H -5.8792986 -1.1614303 1.5186093

H -5.1290029 -1.9353393 2.9163833

Complex 7 (triplet; ppy-localized)

Ir $-0.0618824-0.1113139-0.0348643$

C - 2.65945040 .15571911 .3516818

C $-2.9471454-0.52669400 .1604627$

C $-4.2359733-0.9863509-0.2751463$

C $-4.4405636-1.6548420-1.4511643$

C $-3.3191365-1.9112540-2.2765052$

C - $2.0332394-1.5104720-1.9369093$

C $0.4458596-1.88002890 .7781047$

C $1.3324986-2.6655579-0.0239093$

C $1.7217476-2.0865080-1.3056512$

C $2.5971056-2.6445339-2.2555532$

C $2.8796357-1.9560299-3.4327523$

C $2.2885867-0.7078909-3.6634692$

C $1.4295076-0.1911980-2.7004082$

N $1.1473986-0.8586389-1.5562742$

N 1.62795061 .00882700 .6346808

C 1.68841362 .31126990 .2346257

C $0.62023562 .8450710-0.5688813$

C $0.55225764 .1801621-0.9966572$

C $-0.53494344 .5927879-1.7621363$

C $-1.53925143 .6685631-2.0921664$

C - $1.41851542 .3552801-1.6448453$

$\mathrm{N}-0.36254441 .9450790-0.9018953$

H -5.4303845 -1.9817800 -1.7400393

H - $1.2178234-1.7505769-2.6080312$

C -3.56576740.5405440 2.3986517

C -3.11505641 .19874513 .5148128$

C -1.71800441 .49897313 .6388818$

C -0.84745941 .12284502 .6234728$

H 0.21554161 .31531402 .6879647

H -3.8038205 1.48311914 .3017169

H -2.3988103 3.9596011 -2.6835672

$\mathrm{H}-0.60585145 .6213810-2.0988523$

H $1.35178664 .8525469-0.7119713$

H -2.1577815 $1.5987061-1.8746273$

N 2.80539952 .92982500 .6900687

C 3.43273151 .93422311 .3925837

N 2.75060260 .74841411 .3840488

H $2.4871067-0.1443590-4.5666513$

H $3.5558015-2.3878580-4.1622295$

H $3.0449425-3.6053660-2.0558452$

H $0.94580160 .7674081-2.8227634$ 
C $1.7570336-3.90363010 .4775447$

C $1.3649596-4.40231971 .7091218$

C $0.5025206-3.60705002 .4603546$

C $0.0363026-2.37288892 .0277367$

H $-0.6292294-1.80815802 .6659656$

H $1.7151656-5.36076002 .0642377$

$\mathrm{H}-4.61059940 .29114212 .2845088$

H - 1.32966842 .00620004 .5131846

C $-1.8010685-0.8180870-0.7398752$

$\mathrm{N}-1.26217850 .48109911 .5078537$

C 4.73132242 .10222912 .1025217

H 5.52878672 .38251201 .4045658

H 4.66854152 .89291112 .8589757

H 5.00945771 .16714102 .5942288

F $0.0933296-4.08572703 .6988097$

F $2.6211797-4.7087629-0.2750543$

F -3.5451244 -2.5883649 -3.4724302

F $-5.3582372-0.74063790 .5297588$

Complex 7 (triplet; ptpy-localized)

Ir $0.07255560 .0535728-0.0044848$

C $2.5426796-0.79691421 .3655351$

C $2.9899486-0.15756020 .1291102$

C $4.3238717-0.0177272-0.2819098$

C $4.69890460 .6134848-1.4570748$

C $3.66984571 .1264218-2.2425958$

C $2.32712251 .0250408-1.8988419$

C 0.01144961 .92241680 .7813352

C $-0.67143442 .8773807-0.0360388$

C $-1.17885442 .3786577-1.3093149$

C $-1.87658133 .1107827-2.2888648$

C $-2.28856532 .4897898-3.4647449$

C $-2.00523131 .1330558-3.6679398$

C $-1.31674540 .4422628-2.6784109$

$\mathrm{N}-0.91286141 .0426938-1.5316469$

$\mathrm{N}-1.7780633-0.58307320 .7288932$

C - $2.2097575-1.88956010 .2683362$

C $-1.3675844-2.6102172-0.5655058$

C $-1.6371443-3.9173960-1.0818738$

C $-0.7109614-4.5557951-1.8675789$

C $0.5295896-3.8860773-2.1575049$

C $0.7596186-2.6079303-1.6516378$

$\mathrm{N}-0.1322884-1.9399902-0.8880179$

H $1.58584961 .4542288-2.5607368$

C $3.3513886-1.37457422 .3612772$

C $2.7698237-1.92067113 .5035561$

C $1.3785456-1.88657923 .6555601$

C $0.6112846-1.31198722 .6487561$

H - $0.4651824-1.24257122 .7100171$

H $3.3966137-2.36291924 .2700680$

H $1.2883327-4.3619560-2.7669629$

H -0.9010043 -5.5462703 -2.2636899 
H - 2.5871324 -4.3717321 -0.8229118

H $1.6831857-2.0830282-1.8667559$

$\mathrm{N}-3.4537364-2.17035330 .7711152$

C - $3.7579183-1.06401121 .4971232$

$\mathrm{N}-2.7696234-0.07186821 .4971782$

H -2.3106193 $0.6184158-4.5705086$

H - $2.82542343 .0581728-4.2162897$

H - $2.08471154 .1543838-2.1124419$

H - $1.0709183-0.6055882-2.7722877$

C -0.79862244 .19239990 .4360022$

C - 0.29165844 .61931101 .6515451

C 0.37362763 .66392172 .4158491

C 0.53708762 .34491882 .0131451

H 1.06362161 .66341692 .6681961

H -0.40852545 .64068571 .9834542$

H $4.4219547-1.38186322 .2288753$

H $0.8936156-2.29284234 .5344461$

C $1.95661760 .3781368-0.7063318$

N $1.1728836-0.78838221 .5312582$

F - $1.46558745 .1559406-0.3339888$

F 0.89755764 .06336083 .6413523

F $5.3624788-0.53345820 .5056632$

H $5.73741780 .7034018-1.7409998$

F $4.01557461 .7662878-3.4284197$

C $-5.0340566-0.87450122 .2263943$

H $-4.9471544-0.05483722 .9424733$

H -5.8457335 -0.6345692 1.5260541

H -5.3193673 -1.7957393 2.7437433 